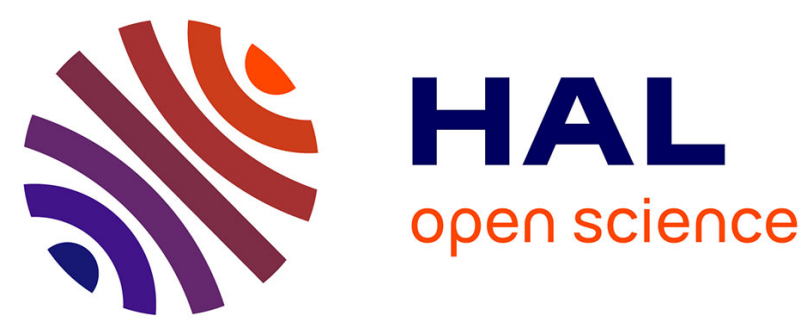

\title{
Composite Nature of Layered Hybrid Perovskites: Assessment on Quantum and Dielectric Confinements and Band Alignment
}

\author{
Boubacar Traoré, Laurent Pedesseau, Linda Assam, Xiaoyang Che, \\ Jean-Christophe Blancon, Hsinhan Tsai, Wanyi Nie, Constantinos C \\ Stoumpos, Mercouri G Kanatzidis, Sergei Tretiak, et al.
}

\section{To cite this version:}

Boubacar Traoré, Laurent Pedesseau, Linda Assam, Xiaoyang Che, Jean-Christophe Blancon, et al.. Composite Nature of Layered Hybrid Perovskites: Assessment on Quantum and Dielectric Confinements and Band Alignment. ACS Nano, 2018, 12 (4), pp.3321-3332. 10.1021/acsnano.7b08202 . hal-01719873

\section{HAL Id: hal-01719873 \\ https://hal.science/hal-01719873}

Submitted on 29 May 2018

HAL is a multi-disciplinary open access archive for the deposit and dissemination of scientific research documents, whether they are published or not. The documents may come from teaching and research institutions in France or abroad, or from public or private research centers.
L'archive ouverte pluridisciplinaire HAL, est destinée au dépôt et à la diffusion de documents scientifiques de niveau recherche, publiés ou non, émanant des établissements d'enseignement et de recherche français ou étrangers, des laboratoires publics ou privés. 


\title{
Composite Nature of Layered Hybrid
}

\section{Perovskites : Assessment on Quantum and \\ Dielectric Confinements and Band Alignment}

\author{
Boubacar Traore, ${ }^{*, \dagger}$ Laurent Pedesseau, ${ }^{\ddagger}$ Linda Assam, ${ }^{ \pm, \Phi}$ Xiaoyang Che, ${ }^{\dagger, \ddagger}$ \\ Jean-Christophe Blancon, ${ }^{\S}$ Hsinhan Tsai, ${ }^{\S}$ Wanyi Nie, ${ }^{\S}$ Constantinos C. \\ Stoumpos," Mercouri G. Kanatzidis," Sergei Tretiak, ${ }^{\S}$ Aditya D. Mohite, ${ }^{\S}$ Jacky \\ Even, ${ }^{\ddagger}$ Mikaël Kepenekian, ${ }^{*} \dagger$ and Claudine Katan ${ }^{* \dagger}$ \\ $\dagger$ Univ. Rennes, ENSCR, INSA Rennes, CNRS, ISCR (Institut des Sciences Chimiques de \\ Rennes) - UMR 6226, F-35000 Rennes, France \\ $\ddagger$ Univ. Rennes, INSA Rennes, CNRS, FOTON (Fonctions Optiques pour les Technologies \\ de l'Information) UMR 6082, F-35000 Rennes, France \\ \TOTAL SA, Tour Coupole, 2 place Jean Miller - La Défense 6 - Courbevoie, France \\ $\S$ Los Alamos National Laboratory, Los Alamos, NM 87545, USA \\ ||Department of Chemistry, Northwestern University, Evanston, IL 60208, USA \\ E-mail: boubacar.traore@univ-rennes1.fr; mikael.kepenekian@univ-rennes1.fr; \\ claudine.katan@univ-rennes1.fr
}




\begin{abstract}
Layered hybrid organic-inorganic perovskites (HOPs) have re-emerged as potential technological solutions for next generation photovoltaic and optoelectronic applications. Their two dimensional (2D) nature confers them a significant flexibility and results in the appearance of quantum and dielectric confinements. Such confinements are at the origin of their fascinating properties and understanding them from a fundamental level is of paramount importance for optimization. Here, we provide an in-depth investigation of band alignments of 2D HOP allowing access to carriers' confinement potentials. 2D HOPs are conceptualized as composite materials in which pseudo inorganic and organic components are defined. In this way, computational modeling of band alignments becomes affordable using first-principles methods. First, we show that the composite approach is suitable to study the position dependent dielectric profiles and enables clear differentiation of the respective contributions of inorganic and organic components. Then we apply the composite approach to a variety of $2 \mathrm{D}$ HOP, assessing the impact on the confinement potentials of well and barrier thickness, of nature of the inorganic well and of structural transitions. Using the deduced potentials, we further discuss the limitations of the effective mass approximation, scrutinizing the electronic properties of this family of composite materials. Our simulations demonstrate type-I dominant band alignment in 2D HOP. Finally, we outline design principles on band alignment towards achieving specific optoelectronic properties. Thus, we present alternative theoretical methods to inspect the properties of $2 \mathrm{D}$ hybrid perovskites and expect that the composite approach will be applicable to other classes of layered materials.
\end{abstract}

\title{
Keywords
}

Band alignment, type-I, quantum confinement, dielectric confinement, composite, photovoltaics, ligth emitters

The rapid rise of hybrid organic-inorganic perovskites' (HOP) power conversion efficiencies $^{1-6}$ over the past few years has placed them as feasible technological solutions for 

this family of materials. ${ }^{7,8}$ Thin films of these materials can be prepared using low-cost solution-based growth methods. However, to bridge the gap from a laboratory characterized material to a technological viable solution compliant with industrial standards, HOP must be resistant to lighting, moisture and humidity stress conditions. The latter proved to be detrimental to 3D perovskites. ${ }^{9-12}$ Recently, layered perovskites have re-emerged as promising alternatives to their $3 \mathrm{D}$ counterparts due to their ability to overcome the above-mentioned mechanisms. ${ }^{5,13,14}$ Besides, the strong vertical orientation of the layers with respect to the substrate and the self-assembly of organic molecules separating the inorganic sheets in layered HOP offers many opportunities for material tuning targeting specific properties via for instance chemical substitution of organic moieties, ${ }^{15,16}$ increasing the length of the inorganic layer ${ }^{17,18}$ or metal substitution in the inorganic framework. ${ }^{19}$ Furthermore, they present attractive optical properties related to large exciton binding energies, ${ }^{20,21}$ promising light emission characteristics ${ }^{22-24}$ and multiexcitonic resonances. ${ }^{25,26}$ Achieving such optimization towards efficient and performing optoelectronic devices requires a thorough understanding of their fundamental photophysical and electronic properties. ${ }^{27}$

An important criterion for the design of efficient solar or light emitting devices is the band alignment of their constituent materials ${ }^{28,29}$ allowing access to carriers' confinement potentials. However, accessing band lineups is not straightforward both from experimental and theoretical perspectives. ${ }^{30}$ Electron affinity models have traditionally been used to align band profiles of heterostructures. ${ }^{31}$ It relies on the affinity of the respective bulk components with the main inconvenient that interface related effects on band offsets are ignored. To calculate band profiles in semiconductors, the transitivity rule has also been used. Here, the band lineup of a heterostructure $\mathrm{A} / \mathrm{C}$ (consisting of materials $\mathrm{A}$ and $\mathrm{C}$ ) is deduced from those of $\mathrm{A} / \mathrm{B}$ and $\mathrm{B} / \mathrm{C}$ separately. ${ }^{32,33}$ First principles based approaches with self-consistent 
charge densities and potentials have also been suggested for that purpose. ${ }^{34,35}$ The direct alignment of band edges cannot be deduced from the first-principles studies of the separate bulk materials due the lack of an intrinsic energy scale, ${ }^{36,37}$ but interface related effects can be accounted for in heterostructures with short periods.

The self-assembled alternating organic and inorganic sheets of layered HOP is reminiscent of an heterostructure. Mitzi and coworkers proposed a type-I quantum-well (QW) picture (Figure 1a), with organic barriers separating inorganic wells. ${ }^{38}$ This idea was verified later for $\left(\mathrm{C}_{10} \mathrm{H}_{21} \mathrm{NH}_{3}\right)_{2} \mathrm{PbI}_{4}$ material ${ }^{39}$ where band alignment and confinement potentials for holes and electrons were deduced based on first-principles calculations. In the field of conventional semiconductors, further modeling of heterostructures is performed using such confinement potentials in empirical Hamiltonians. For instance, in a quantum well model, confinement potentials can be used to calculate carriers' densities ${ }^{40}$ and subsequently their mobilities, quantities of significant importance in device operation. The applicability of this method to layered HOP still requires detailed investigation, especially on how materials' structure and composition affect band alignments.

In this work, we introduce the foundation of the composite approach and subsequently demonstrate, by means of first-principles calculations, that it applies to a wide range of layered hybrid organic-inorganic perovskites. It provides fruitful insights for inspecting quantum and dielectric confinement effects. First, we show that high-frequency dielectric constant profiles, related to dielectric confinement, exhibit a composite behavior. Next, we perform a detailed investigation of band alignment and confinement potentials within the framework of our composite approach considering sequentially the effects of: (i) the composition of the inorganic well and organic barrier (ii) the thickness of the molecular barriers and phase transitions, and (iii) the thickness of the perovskite layers. The later case is then used to inspect the validity of the effective mass approximation in layered HOP. We demonstrate that Type-I confinement is the dominant band alignment in 2D HOP. Before concluding, material design principles are outlined for achieving specific optoelectronic properties. We believe that our 
a)

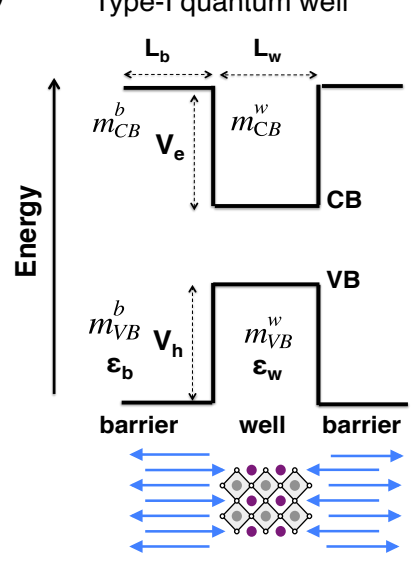

Possible type-Il quantum well

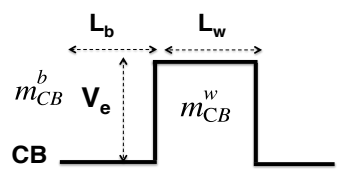

vs.

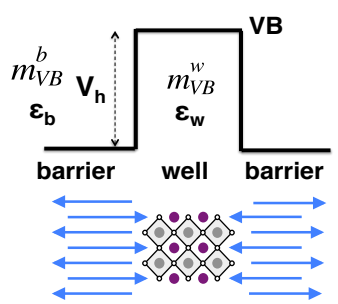

b)

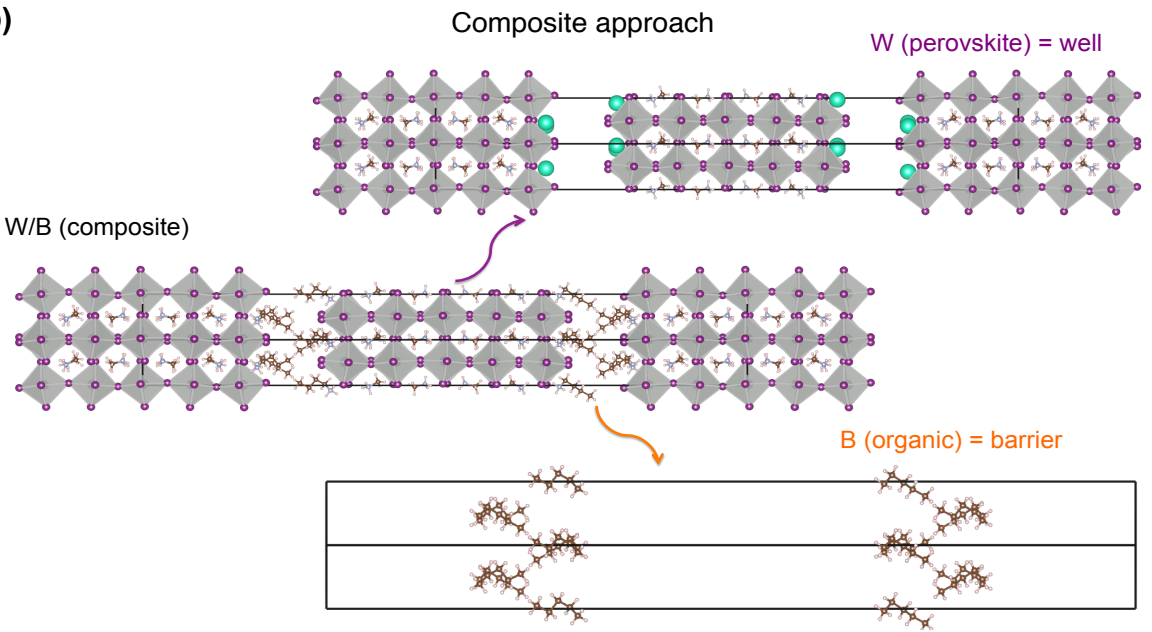

Figure 1: Composite approach to layered hybrid perovskites. (a) schematics of type-I ${ }^{38}$ and possible type-II quantum wells. VB and CB correspond to valence and conduction bands, respectively. Note that, for a type-II quantum well, VB and $\mathrm{CB}$ are not spatially localized in the same layer. L, m, $\epsilon$ and V stand for thickness, effective mass, dielectric constant and confinement potential, respectively. Subscripts and superscripts b, w, e and h denote barrier, well, electron and hole, respectively. The schematics structure below shows the definition of the well and the barrier regions in a pictorial perovskite structure (arrows correspond to the barrier and corner shared octahedra to the well). (b) General presentation of the composite approach: the real composite material W/B is decomposed into two parts: the perovskite well section $\mathrm{W}$ where the organic molecules of the barrier are replaced by $\mathrm{Cs}^{+}$atoms and the organic barrier section $\mathrm{B}$, in which $\mathrm{NH}_{3}^{+}$are replaced by $\mathrm{CH}_{3}$ groups. 
composite approach will be applicable to other classes of 2D hybrid materials, where organic and inorganic components coexist.

\section{Results/Discussion}

\section{Concept of the Composite approach for dielectric and quantum con- finements in layered HOP}

We start by defining the composite approach for layered HOP. In a layered HOP, the organic molecules exhibit a wider band gap as compared to the inorganic perovskite layer. Such structure can be viewed as a type-I quantum well ${ }^{38}$ (Figure 1a). In this configuration, the organic molecules and inorganic (perovskite) layers correspond to the barrier and the well of the potential, respectively. Hence, in the composite approach, the quantum well like structure of layered HOP is a heterostructure ${ }^{39}$ where we define three model systems (Figure 1b). For the proof of concept and without loss of generality, we use $(\mathrm{BA})_{2}(\mathrm{MA})_{4} \mathrm{~Pb}_{5} \mathrm{I}_{16}(\mathrm{BA}=$ $\left.\mathrm{CH}_{3}\left(\mathrm{CH}_{2}\right)_{3} \mathrm{NH}_{3}\right)$ member of Ruddelsden-Popper (RP) phases type of layered perovskites. ${ }^{18}$ In the figure, $\mathrm{W} / \mathrm{B}$ corresponds to the complete crystallographic structure. ${ }^{18}$ Although, we rely on the experimental structures, the method is applicable to DFT based optimized geometries as well. Structure $\mathrm{W}\left(\mathrm{Cs}_{2}(\mathrm{MA})_{4} \mathrm{~Pb}_{5} \mathrm{I}_{16}\right)$ is the five perovskite layers of W/B with the inter-layer molecules BA replaced by Cs. The validity of such Cs substitution is discussed in the supplementary information (Figure S1). Structure B consists of the inter-layer organic molecules $\left(\mathrm{CH}_{3}\left(\mathrm{CH}_{2}\right)_{3} \mathrm{CH}_{3}\right)_{2}$ where the $\mathrm{NH}_{3}^{+}$group of the $\mathrm{BA}$ cation is substituted by the $\mathrm{CH}_{3}$ moiety. In both $\mathrm{W}$ and $\mathrm{B}$ structures, the electroneutrality of the system is enforced. Next, we inspect the dielectric profiles and band alignments within the composite method using $(\mathrm{BA})_{2}(\mathrm{MA})_{4} \mathrm{~Pb}_{5} \mathrm{I}_{16}$ as a model system.

Layered perovskites have large exciton binding energies due to the dielectric and quantum confinement effects. ${ }^{41,42}$ The barrier dielectric constant is usually much smaller than that of the inorganic well. Consequently, the effective electron-hole Coulomb interaction is enhanced 

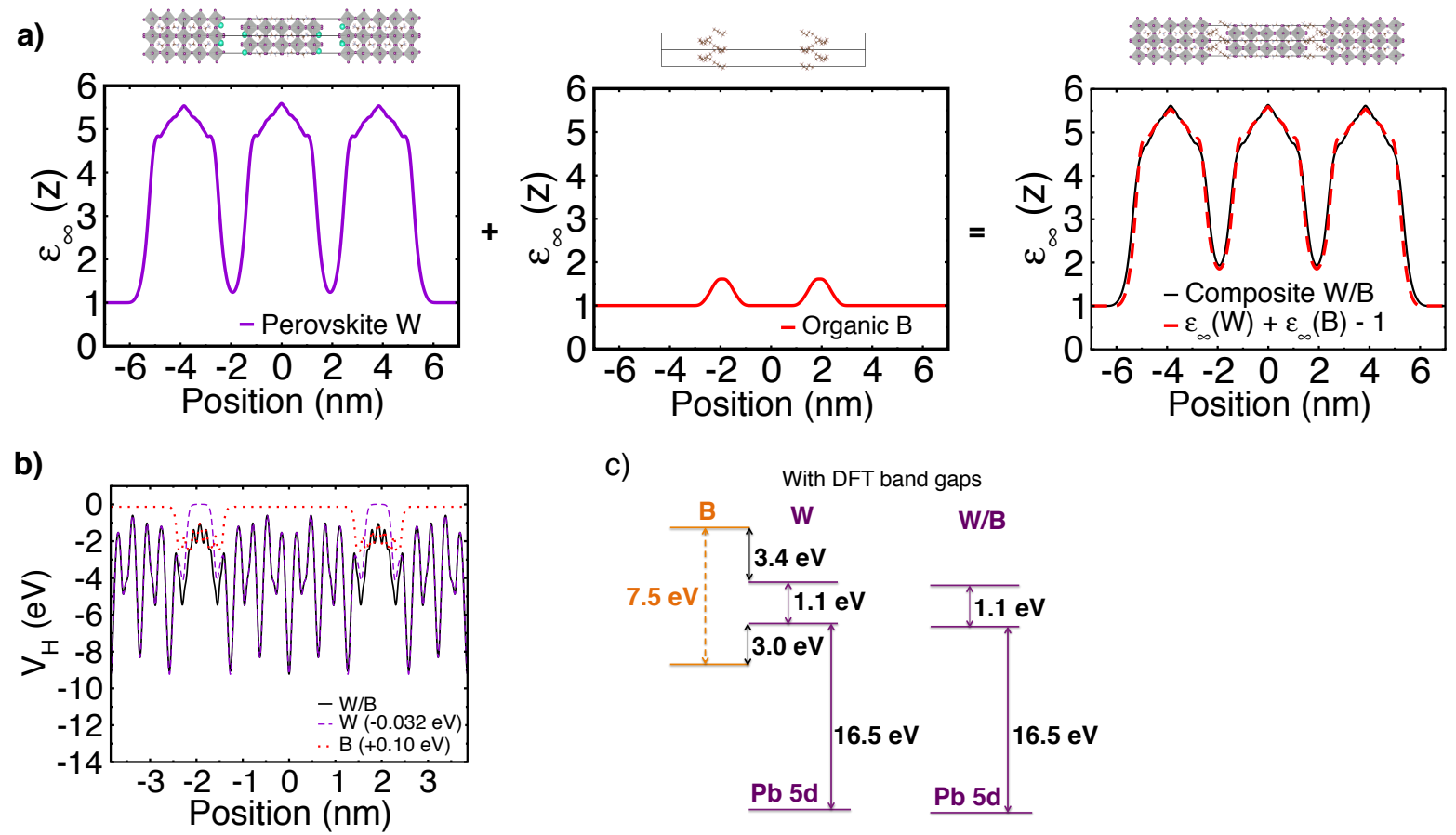

Figure 2: Composite approach to dielectric profiles and band alignment. (a) Dielectric profiles computed for each of the sections $\mathrm{W}$ and $\mathrm{B}$ of the layered hybrid perovskite $(\mathrm{BA})_{2}(\mathrm{MA})_{4} \mathrm{~Pb}_{5} \mathrm{I}_{16}{ }^{18}$ from Figure 1b. The dielectric profile of the real composite structure $\mathrm{W} / \mathrm{B}$ computed for $(\mathrm{BA})_{2}(\mathrm{MA})_{4} \mathrm{~Pb}_{5} \mathrm{I}_{16}$ is also compared to the sum of the individual components $\mathrm{W}$ and $\mathrm{B}$. It reveals the composite nature of the dielectric profiles for the 5-octahedron thick layered perovskite. b) Hartree potential profiles $\left(\mathrm{V}_{H}\right)$ obtained for the structures of Figure 1b. This affords potential shifts needed to align $\mathrm{V}_{H}$ of the individual components $\mathrm{W}$ and $\mathrm{B}$ to that of the real composite W/B. c) Band alignment between $\mathrm{W}$ and $\mathrm{B}$ for $(\mathrm{BA})_{2}(\mathrm{MA})_{4} \mathrm{~Pb}_{5} \mathrm{I}_{16}$ obtained considering the $\mathrm{V}_{H}$ shifts deduced in panel b. Noteworthy, for the perovskite layer, the shift in $\mathrm{V}_{H}$ between $\mathrm{W} / \mathrm{B}$ and $\mathrm{W}$ is equal to that of $\mathrm{Pb} 5 \mathrm{~d}$ states between $\mathrm{W} / \mathrm{B}$ and $\mathrm{W}$. 
in the well. The image-charge method assuming abrupt interface between the barrier and the well has often been used to account for electron-hole Coulomb interaction in the two particle empirical Hamiltonian. ${ }^{42}$ First principles methods including interface related effects in dielectric profiles have also been developped. ${ }^{43,44}$ Subsequently, we apply these methods to hybrid organic-inorganic perovskites to account for dielectric confinement effects. ${ }^{45,46}$ To show the composite behavior of dielectric profiles in these layered perovskites, we calculate the position dependent high frequency dielectric constant along the stacking axis in the composite $\mathrm{W} / \mathrm{B}$, the perovskite well $\mathrm{W}$ and the organic barrier B (Figure 2a). $\epsilon_{\infty}$ varies from 1.9 in the organic barrier B to 5.5 in the perovskite well W, clearly highlighting the dielectric confinement. In the perovskite layer $\mathrm{W}, \epsilon_{\infty}$ reaches that of bulk $3 \mathrm{D} \mathrm{MAPbI}_{3},{ }^{18}$ and is in a reasonable agreement with the experimental 6.5 value of the latter. ${ }^{47}$ Figure 2a compares the dielectric profile of $\mathrm{W} / \mathrm{B}$ and the addition of those of $\mathrm{W}$ and $\mathrm{B}$. The two dielectric profiles are superimposed using:

$$
\epsilon_{\infty}^{W / B}(z)=\epsilon_{\infty}^{W}(z)+\epsilon_{\infty}^{B}(z)-1
$$

where -1 is used to avoid the double counting of the contribution of the vacuum to the dielectric constant. Equation 1 can be interpreted as the sum of the induced polarizations due to structures $\mathrm{W}$ and $\mathrm{B}$ and from which the equivalent electric susceptibility $\chi(\chi=\epsilon-$ 1) for the composite gives:

$$
\chi_{W / B}(z)=\chi_{W}(z)+\chi_{B}(z) .
$$

Hence, using the composite approach, the dielectric profile accounting for the sole electronic contributions (optical dielectric constant profile) of the complete structure can be viewed as the sum of the contributions of the constituting components of the whole structure. The computation of the dielectric profiles presented in this work is performed only for the dielectric component along the stacking axis. It allows identifying clearly the contribution of the respective layers to the total dielectric profile in the composite approach. We 
further checked that the composite approach towards the high-frequency dielectric constant also applies in the plane of the quantum well and that the dielectric constant is anisotropic in these structures. Additionally, for thin inorganic layers especially $n=1$, the dielectric profiles of the composite and the addition of the constituting parts do not perfectly match and the error is about $7 \%$ (Figure S2). This error becomes negligible with thicker perovskite layers (Figure S2). This indicates that $\mathrm{n}=1$, consisting of only one inorganic octahedron, is not a true composite and the relative large error suggests that in this limiting case the properties are influenced by interface effects.

In hybrid perovskites, electron-phonon coupling becomes significant in the THz region which also gets manifest in the dielectric response of the structures. ${ }^{4-50}$ Such an effect of polar phonons on the dielectric constant can, in principle, be accounted for computationally through the relaxation of both the electronic density and the atomic positions under an external applied electric field. ${ }^{46}$ However, the relaxation of the perovskite $\mathrm{W}$ layer alone with $\mathrm{Cs}^{+}$substitution or the organic barrier B alone is not relevant due to the strong influence of barrier-perovskite interactions on the relaxation of the composite $\mathrm{W} / \mathrm{B}$. The same conclusion can be drawn for the contribution of the stochastic motions of molecular cations to the lowfrequency dielectric constant. ${ }^{7}$ Besides, the perovskite and barrier layers in the composite method are mere conceptual structures and are not suitable for structural optimization.

The computed high frequency dielectric constant profiles involve the relaxation of the sole electronic density under an applied external field along the stacking axis. This electronic density involves the valence band (VB) states (occupied states) as the conduction bands are completely unoccupied in these undoped semiconductor materials. We expect that the composite method will permit the evaluation of carrier's confinement potentials, particularly relevant to the VB. We show next its applicability to compute band alignment with the subsequent deduction of relevant confinement potentials.

The evaluation of band alignment in a heterostructure using either electron affinity rule or first principles based DFT methods requires information on its bulk materials. In the 
former, alignment is achieved through the affinities of the respective bulk materials; in the latter, band edge energies are referenced to the Hartree potentials obtained from the bulk components. However, bulk structures for the perovskite and organic parts of layered HOP cannot be defined as in classical semiconductor heterostructures. ${ }^{39}$ This is possible, however, in the present composite approach where the band alignment is carried out through the Hartree potential profiles. The latter are calculated from DFT simulations of three different structures (W/B, W, and B). This is illustrated for $(\mathrm{BA})_{2}(\mathrm{MA})_{4} \mathrm{~Pb}_{5} \mathrm{I}_{16}$ in Figure $2 \mathrm{~b}$. Hartree potentials are then obtained by averaging the potentials in the plane perpendicular to the stacking axis of the layered structure. Shifting the potential profile of the perovskite layer $\mathrm{W}$ with $\mathrm{Cs}^{+}$substitution $(-0.032 \mathrm{eV}$ for our example) results in a perfect match to that of the perovskite part of the whole $\mathrm{W} / \mathrm{B}$ structure. Thus the electronic states of the inorganic layer and close to the band gap edges are fairly well described by the $\mathrm{W}$ model structure, otherwise such a match would not be possible. Similarly, a shift ( $+0.10 \mathrm{eV}$ for our example) of the potential profile of the organic structure B results in a perfect match with that of the organic part of $\mathrm{W} / \mathrm{B}$. This procedure demonstrates that, with regards to band alignment, layered perovskites can be considered as composite materials with weak interactions between the organic and inorganic layers. ${ }^{39}$

From the Hartree potentials alignment, we further deduce an appropriate band lineup between the perovskite and organic layers of the 2D material. The procedure consists of applying the potential shifts obtained from $\mathrm{V}_{H}$ alignment to corresponding $\mathrm{VB}$ of $\mathrm{W}$ and $\mathrm{B}$ structures. For our exemple, a downward shift of $0.032 \mathrm{eV}$ (as obtained from Figure 2b) is applied to VB of the perovskite part $\mathrm{W}$ to match it to that of the structure $\mathrm{W} / \mathrm{B}$ as shown in Figure 2c. Notably, the very same shift $(-0.032 \mathrm{eV})$ is derived to align the $\mathrm{Pb} 5 \mathrm{~d}$ states of $\mathrm{W}$ to those of $\mathrm{W} / \mathrm{B}$. Indeed $\mathrm{Pb} 5 \mathrm{~d}$ states are low-lying orbitals unaffected by the chemical substitution for states close to the electronic gap and could be used as electronic markers in $\mathrm{Pb}$ based perovskites. ${ }^{51}$ To obtain the level of $\mathrm{Pb}-5 \mathrm{~d}$ states, we use orbital resolved projected density of states. The fluctuations observed between the different $5 \mathrm{~d}$ orbital entries (vide 
infra) are related to the chemical environment around $\mathrm{Pb}$. As the slight difference with Ref. 51, it may essentially be due to the choice of the basis-set and pseudo-potentials adopted for those calculations. A similar procedure can be applied to systems with a different chemical composition in which case low-lying orbital entries could be used as markers (for example, 4d states for Sn based 2D HOP, vide infra). The alignment of structure B is deduced by applying an upward shift of $0.10 \mathrm{eV}$ to its $\mathrm{VB}$ as obtained from Hartree potentials alignment of Figure 2b. Hence, using the composite approach of 2D HOP, we afford a complete band alignment of the system and shows the type-I nature of confinement for this structure.

Although conduction band (CB) lineup is also shown in Figure 2c, we stress that this approach is more relevant to $\mathrm{VB}$ alignment as the polarized electron gas is related to the occupied states only. Furthermore, due to the underestimation of the electronic band gap by DFT, it does not allow a precise alignment of CBs. Such treatment would require the use of more sophisticated and computationally demanding methods including both many-body corrections and spin-orbit coupling interactions. ${ }^{51,52}$ Thus, whenever possible we use available experimental band gaps for CB alignment. We remind that these band gaps are usually optical ones that include excitonic ccontributions (Table S6). Such procedure assumes that band gap corrections mostly affect the conduction band. ${ }^{36}$ As such, confinement potentials at $\mathrm{CB}$ may present larger errors. In the following, we apply the composite approach to a wide range of layered HOP and assess various effects on the confinement potentials.

\section{Chemical tuning of the inorganic well}

Because band edge states (valence band maximum (VBM) and conduction band minimum $(\mathrm{CBM}))$ in $\mathrm{HOP}$ are mainly determined by the hybridization between metal and halogen orbitals, substituting halogen atoms as well as mixing them have been used to tailor the band gap and photoluminescence (PL) properties of these materials. ${ }^{53,54}$ For instance, in $(\mathrm{EA})_{4} \mathrm{~Pb}_{3} \mathrm{X}_{10}\left(\mathrm{EA}=\mathrm{CH}_{3} \mathrm{CH}_{2} \mathrm{NH}_{3}\right)$ layered perovskites, an increase of the band gap was observed in the absorption or emission spectra when $\mathrm{X}$ was varied from $\mathrm{Br}$ to $\mathrm{Cl}^{24} \mathrm{We}$ inspect 
the effect of such change of the halogen atom on band alignment with the phenyl methylammonium lead halide $(\mathrm{PMA})_{2} \mathrm{PbX}_{4}\left(\mathrm{PMA}=\mathrm{C}_{6} \mathrm{H}_{5} \mathrm{CH}_{2} \mathrm{NH}_{3}\right)$ series of layered perovskites (Figure 3a). Here, DFT band gaps are used for CB alignment since experimental data are not available for the entire series. Figure 3b presents their deduced type-I band alignments considering the experimental structures (Figure $3(\mathrm{a})$ ) for $\mathrm{X}=\mathrm{I},{ }^{55} \mathrm{Br}^{15}$ and $\mathrm{Cl} .{ }^{56}$ The DFT band gap increases with lighter halogen atoms whereas the in-plane lattice constants decrease in the same series. This stabilizes (destabilizes) Pb-halogen hybridization at VBM (CBM) and results in the band gap increase for lighter halogens. Incidentally, significant structural

a)

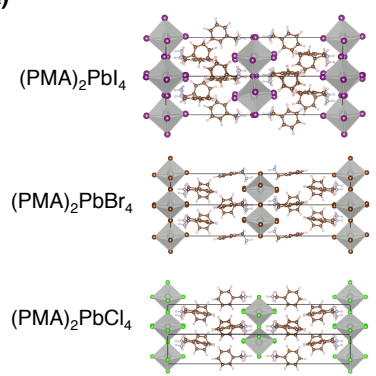

d)

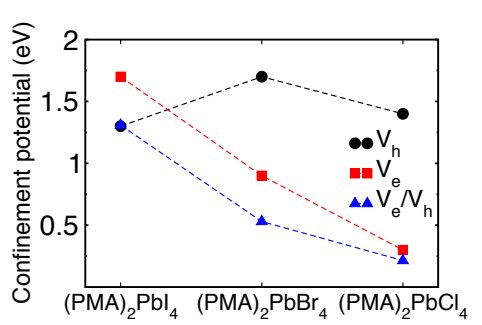

b)

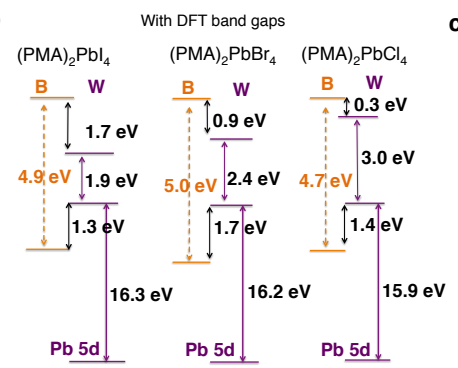

c) With experimental band gaps

$(\mathrm{PMA})_{2} \mathrm{PbCl}_{4}$

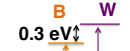

$\frac{0.30}{\hat{i}}$
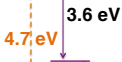

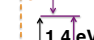

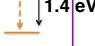

$\mathrm{Pb} \underline{5 \mathrm{~d}} \coprod^{15.9 \mathrm{eV}}$

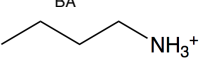

e)
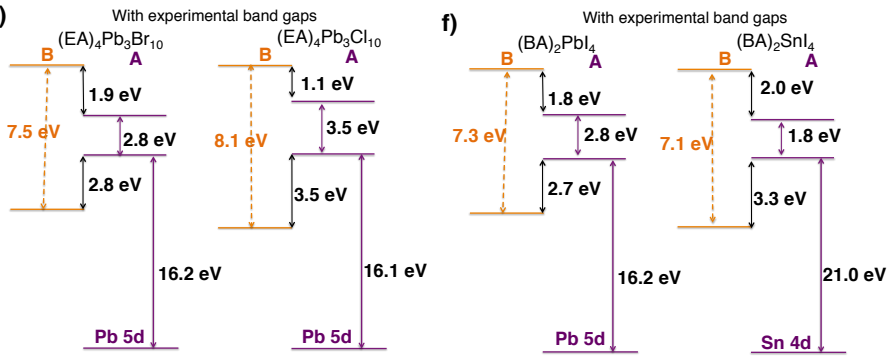

Figure 3: Effect of the chemical composition of the perovskite well on band alignments. (a) Experimental structures used for $(\mathrm{PMA})_{2} \mathrm{PbX}_{4}$ series to investigate the effect of halogen atom on band alignment. $\mathrm{X}$ refers to the halogen ( $\mathrm{I}, \mathrm{Br}$ or $\mathrm{Cl}$ ); and schematics of the corresponding molecular barriers considered in this panel. PMA for phenyl methylammonium, EA for ethylammonium and BA for butylammonium. (b) Computed band alignments of $(\mathrm{PMA})_{2} \mathrm{PbX}_{4}$ and corresponding band offsets. Since, we do not have the experimental band gaps for all the compositions, DFT band gaps are used for conduction band lineup. For $\mathrm{X}=\mathrm{Cl}$, the band alignment tends to deviate from type-I. (c) Same as panel b considering the experimental optical band gap for $\left(\mathrm{PMA}_{2} \mathrm{PbCl}_{4}\right.$, showing the possibility of a type-II band alignment. (d) Variation of hole (circle) and electron (square) confinement potentials extracted from panel $\mathrm{b}$ and corresponding ratios. (e) Band alignment computed for (EA) ${ }_{4} \mathrm{~Pb}_{3} \mathrm{X}_{10}$ using experimental optical band gaps ${ }^{24}$ to align CBM (for alignments with DFT band gaps see Figure S4). (f) Band alignment showing the effect of metal substitution $(\mathrm{BA})_{2} \mathrm{MI}_{4}(\mathrm{M}=$ $\mathrm{Pb}, \mathrm{Sn}$ ) on confinement potentials. Experimental optical band gaps are used for CB alignment(see Figure S5 for DFT band gaps). 
distortions occur, consistent with noticeable octahedral tiltings $(\beta, \delta)$ induced in the inorganic layer when the halogen is varied from I to $\mathrm{Cl}$. The tiltings $(\beta, \delta)$ are defined in Figure S3 and their values are reported in Table S1. Such distortions affect more the VB than the CB since the former consists of an antibonding hybridization between halogen $\mathrm{X}$ (p states) and lead $\mathrm{Pb}$ (s states); and the latter composes a bonding hybridization between $\mathrm{Pb}$ ( $\mathrm{p}$ states). One would then expect that a pronounced effect on VB states would significantly affect hole confinements from I to $\mathrm{Cl}$. However, we notice that the hole confinement potentials $\left(\mathrm{V}_{h}\right)$ have similar values for the different (PMA) ${ }_{2} \mathrm{PbX}_{4}$ wells (Figure $3 \mathrm{~d}$ ). The $0.3 \mathrm{eV}$ difference of $\mathrm{V}_{h}$ between $\mathrm{Br}$ based well as compared to that of $\mathrm{Cl}$ falls within the uncertainties in the DFT band gaps of the organic and inorganic sheets. At first, the electron confinement potential $\left(\mathrm{V}_{e}\right)$ for $(\mathrm{PMA})_{2} \mathrm{PbI}_{4}$ appears larger than $\mathrm{V}_{h}$ considering DFT band gaps. This conclusion is however expected to be revised using experimental band gaps or under accurate band gap correction via sophisticated many body correction schemes. Noteworthy, despite systematic errors in the band gap values, $(\mathrm{PMA})_{2} \mathrm{PbBr}_{4}$ and $(\mathrm{PMA})_{2} \mathrm{PbCl}_{4}$ present much lower $\mathrm{V}_{e}$ as compared to $\mathrm{V}_{h}$. This trend suggests that the confinement type for (PMA) ${ }_{2} \mathrm{PbX}_{4}$ compounds may undergo a crossover from type-I to type-II under a proper band gap correction. For instance, considering the experimental $3.65 \mathrm{eV}$ optical band gap of $(\mathrm{PMA})_{2} \mathrm{PbCl}_{4},{ }^{56}$ the band alignment of (PMA $)_{2} \mathrm{PbCl}_{4}$ may change from type-I to a type-II confinement (Figure 3c). Although such a type-II alignment has not yet been reported in layered hybrid perovskites, an experimental measurement aiming at probing the lifetime of excitons could be decisive about its possible existence. From such a spectroscopic measurement, longer lived indirect excitons could point to a type-II band heterostructure.

We further inspect the effect of halogen substitution considering $(\mathrm{EA})_{4} \mathrm{~Pb}_{3} \mathrm{X}_{10}$ type of perovskites ${ }^{24}$ where $\mathrm{X}$ represents $\mathrm{Br}$ and $\mathrm{Cl}\left(\mathrm{EA}=\mathrm{CH}_{3} \mathrm{CH}_{2} \mathrm{NH}_{3}\right)$. The corresponding band alignments showing the relevant confinement potentials are shown in Figure 3e. We notice a similar trend of higher $\mathrm{V}_{h}$ as compared to $\mathrm{V}_{e}$. Notably, the decrease of $\mathrm{V}_{e}$ in $(\mathrm{EA})_{4} \mathrm{~Pb}_{3} \mathrm{Cl}_{10}$ also points to the less pronounced type-I carrier confinement when $\mathrm{Cl}$ is used as the halogen. 
We finally investigate the effect of metal substitution on band alignment considering $(\mathrm{BA})_{2} \mathrm{MI}_{4}(\mathrm{M}=\mathrm{Pb}, \mathrm{Sn}) .{ }^{57-59}$ Notice that $\mathrm{Sn} 4 \mathrm{~d}$, unaffected low-lying orbitals, were used as electronic markers for $(\mathrm{BA})_{2} \mathrm{SnI}_{4}$ as shown in Figure 3f. $(\mathrm{BA})_{2} \mathrm{SnI}_{4}$ presents both larger hole and electronic confinement potentials as compared to $(\mathrm{BA})_{2} \mathrm{PbI}_{4}$ probably due to the narrower band gap of $(\mathrm{BA})_{2} \mathrm{SnI}_{4}$.

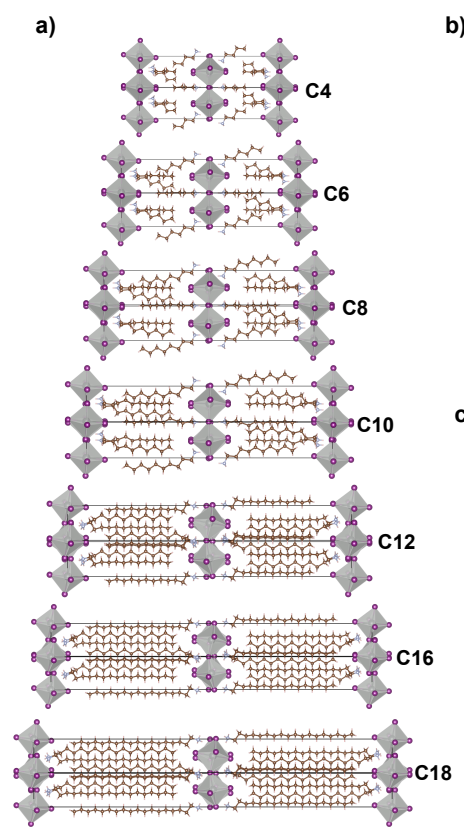

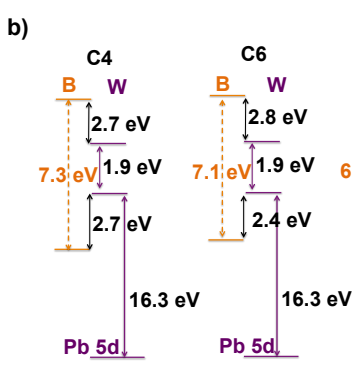

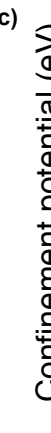

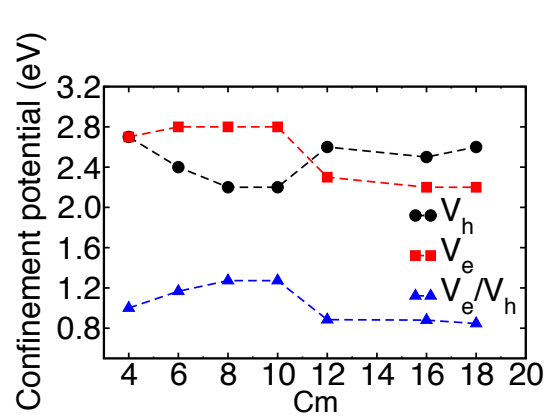

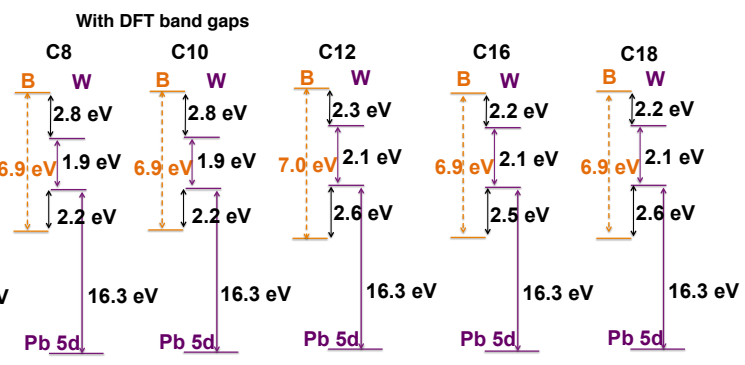

d)

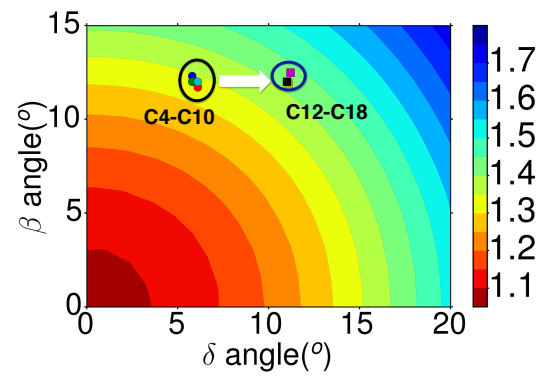

Figure 4: Band alignement for various barrier thicknesses. (a) Structures of $\left(\mathrm{C}_{m} \mathrm{H}_{2 m+1} \mathrm{NH}_{3}\right)_{2} \mathrm{PbI}_{4}$. $\mathrm{Cm}$ next to the structures refers to the number $\mathrm{m}$ of carbon atoms in the alkyl chain. (b) Computed band alignments for the alkyl-based structures shown in panel a, taking crystallographic data recorded at room temperature. Since, all the experimental band gaps are not available for the series, we use DFT band gaps for conduction band lineup. (c) Variation of hole (circle) and electron (square) confinement potentials extracted from panel b and corresponding ratios. (d) Computed band gaps plotted on the 2D color map illustrating the variation of the band gap with octahedral in-plane (beta) and out-of-plane (delta) tilt angles for the $\mathrm{Cs}_{2} \mathrm{PbI}_{4}$ model structure (spin-orbit coupling is included). ${ }^{60}$ Tilt angles are summarized in Table S2.

\section{Effect of barrier length}

Yet another attractive feature of $2 \mathrm{D} \mathrm{HOP}$ is the flexibility of the organic barrier and the possibility of tuning its length and chemical composition. To assess the effect of the barrier length on the band lineup, we investigate some members of the alkyl ammonium lead iodide 
$\left(\mathrm{C}_{m} \mathrm{H}_{2 m+1} \mathrm{NH}_{3}\right)_{2} \mathrm{PbI}_{4}$ series of layered $\mathrm{HOP}\left(\mathrm{C}_{m}\right.$ with $\mathrm{m}$ carbons in the barrier molecule), in their orthorhombic phases (Figure 4a, Table S2). ${ }^{57,61,62}$ Figures 4b,c show their band alignments and the variation of the deduced confinement potentials with increasing barrier length, respectively. All plots reveal a type-I alignment. From C4 to C10, $\mathrm{V}_{e}$ is larger than $\mathrm{V}_{h}$ with almost no variation as a function of the barrier length. This behaviour is reversed for the $\mathrm{C} 12-\mathrm{C} 18$ series where $\mathrm{V}_{h}$ is larger than $\mathrm{V}_{e}$ with little dependence on the length of the barrier. Additionally, a band gap increase of about $0.2 \mathrm{eV}$ is also deduced for $\mathrm{C} 12-\mathrm{C} 18$ compounds as compared to C4-C10.

This band gap increase and the associated reverse trend of the confinement potentials can be related to distortions in the structures of $\mathrm{C} 12-\mathrm{C} 18$, as compared to C4-C10. In fact, structural distortions have a significant effect on the band gap of hybrid perovskites with a direct impact on their optical properties. ${ }^{8,27,52,63}$ To rationalize the interplay between octahedral distortions and band gap variation with the associated reversed confinement potentials, we use a $\mathrm{Cs}_{2} \mathrm{PbI}_{4}$ model system from which two characteristic angles $\beta$ (in-plane tilt) and $\delta$ (out-of-plane tilt) can be defined. ${ }^{60}$ The angles deduced from the different experimental structures (Table S2) can then be plotted on the computed 2D map of band gap variations with octahedral tilting, Figure 4d. The entire alkyl series (C4-C18) exhibits similar in-plane distortion $\beta$. However, the out-of-plane tilt angle $\delta$ is larger for C12-C18 as compared to C4-C10 which is accompanied by an increase of the band gap in the 2D map (Figure 4d). ${ }^{60}$ Hence, the band gap increase and the reversed confinement potentials are related to the octahedral distortions that build up when going from C12 to C18. A noticeable change in the in-plane lattice constants also occurs in C12-C18 compared to C4-C10 (Table S2). Thus, increasing the barrier length can result in a change of hole and electron confinement potentials, which in turn can influence the efficiency of carrier transport or confinement in the structure. As such, selecting an appropriate cation length is, among others, a practical way to fine tune the optoelectronic properties of layered perovskites 


\section{Effect of lattice distortion related to structural phase transitions}

For a given composition, structural distortions can also be induced in the inorganic layer due to temperature or pressure driven structural phase transitions. It may have a noticeable influence on the hybridization of the metal-halogen states especially at the valence band, which in turn influences hole confinement. The effect of such phase transitions is inspected with C10, which adapts two orthorhombic structures with space group Pbca, recorded at $268 \mathrm{~K}$ and $293 \mathrm{~K} .{ }^{62}$ Their corresponding confinement potentials are shown in Figure 5a and the relevant band alignment is shown in Figure S6. Here, DFT band gaps are used. The band gap of $\mathrm{C} 10$ increases from $1.9 \mathrm{eV}$ to $2.1 \mathrm{eV}$ by lowering the temperature (Figure S6), in agreement with the structure's phase color change from orange to yellow observed experimentally. ${ }^{62}$ Again, using the 2D map shows that the band gap increase results from larger octahedral tilt angles in the low temperature phase (Table S3, Figure 5b) which leads to the inversion of the respective amplitudes of $\mathrm{V}_{h}$ and $\mathrm{V}_{e}$.

\section{Effect of the perovskite layer thickness}

The self-organized nature of 2D HOP confers them a high degree of flexibility for tuning the size and chemical composition of the constituent layers. An interesting example of such tuning with potential technological promises is the RP series of layered perovskites of general formula $(\mathrm{BA})_{2}(\mathrm{MA})_{n-1} \mathrm{~Pb}_{n} \mathrm{I}_{3 n+1}$. $\mathrm{n}$ corresponds to the number of octahedra in each perovskite layer and defines its thickness (Table S4). ${ }^{5,17,21,57,64}$ We consider n=1-5 (Figure 6a) members of the series and summarize their crystallographic information in Table S4. Figures $6 \mathrm{~b}$ and $\mathrm{c}$ show the band alignments and the variation of the confinement potentials with increasing $\mathrm{n}$ of the perovskite layer, respectively. Here, the experimental continuum band gaps ${ }^{21}$ excluding excitonic contributions are used to align CBM (band alignments with DFT band gaps are provided in Figure S7). For all n, a type-I band lineup is obtained. A larger hole confinement potential $\mathrm{V}_{h}$ is deduced for all $\mathrm{n}$ with $\mathrm{n}=3$ showing the largest 
a)
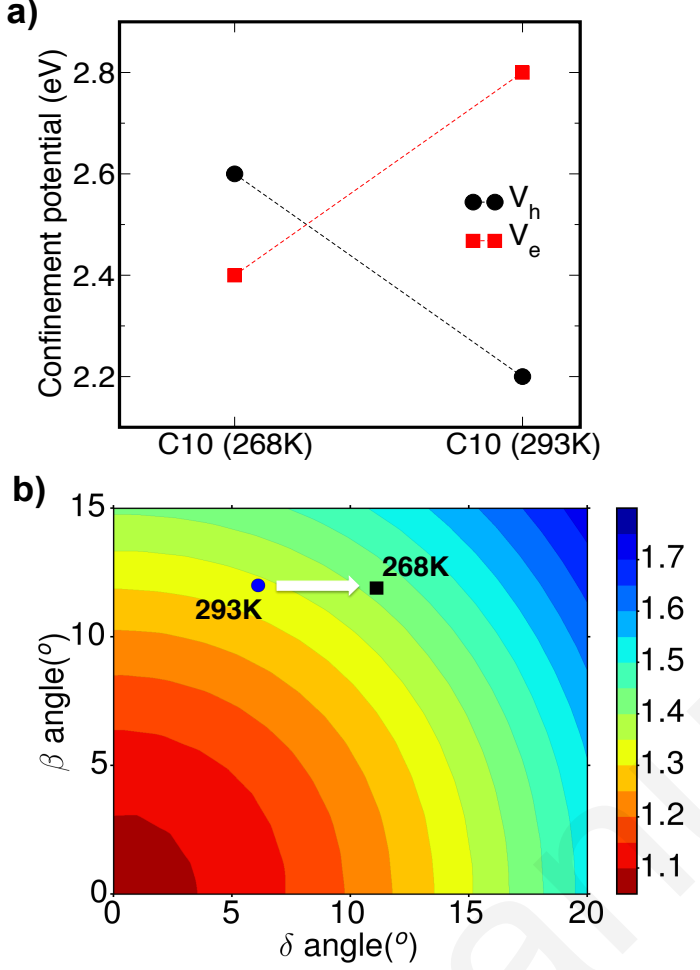

Figure 5: Assessing the impact of phase transition on band alignment. (a) Variation of the confinement potentials with phase transition. C10 (Figure 4a) is used for this example. (b) 2D color map of the electronic band gap variation of $\mathrm{C} 10$ with respect to $(\beta, \delta)$ as a result of phase transition.

$\mathrm{V}_{h^{-}}-\mathrm{V}_{e}$ difference. Noteworthy, the band gap decreases with increasing $\mathrm{n}$ which is a signature of diminishing quantum confinement effect in thicker perovskite layers. ${ }^{18,21}$ Hence, confinement potentials and quantum confinement effects are inter-related in these structures. In the section applying with the composite approach to band alignment, we showed that the shift of $\mathrm{Pb} 5 \mathrm{~d}$ states between the composite and the inorganic layer is the same as their Hartree potentials difference. This does not exactly hold for $n=1$ where the difference between the Hartree potentials and $\mathrm{Pb} 5 \mathrm{~d}$ states amounts to $0.14 \mathrm{eV}$. In general, we obtain 0.1 $\mathrm{eV}$ to $0.15 \mathrm{eV}$ differences for other structures with one inorganic layer (see section $\mathrm{S} 8$ of the supplementary information). A similar conclusion was reached from the study of dielectric profiles, suggesting that the $n=1$ system is influenced by interface effects. Thus, the composite approach is best suited for $n>1$. The effect of well thickness can be further analyzed 
a)

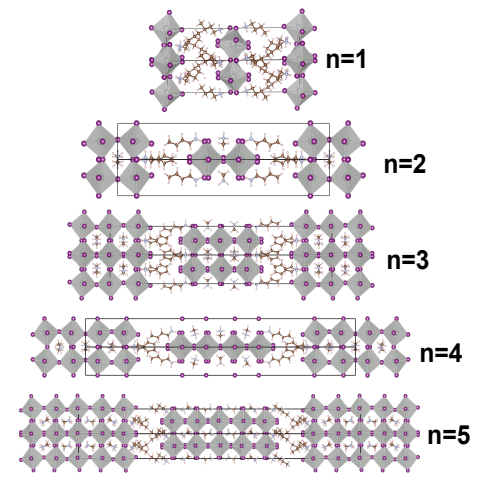

b)

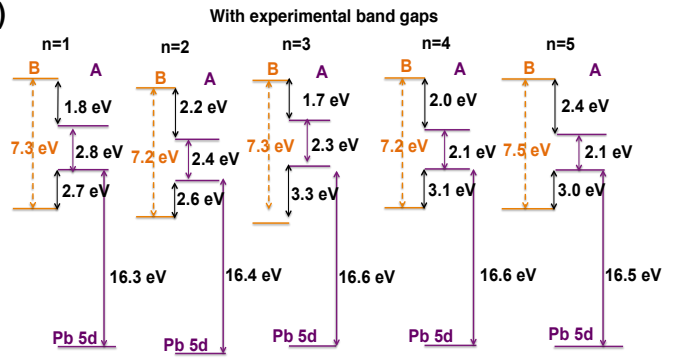

c)

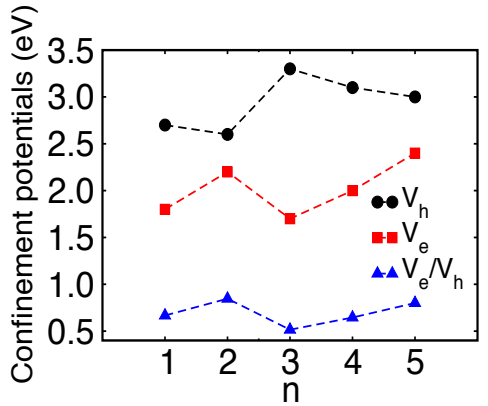

Figure 6: Band alignement for various well thicknesses. (a) Schematics of the HOP structures $(\mathrm{BA})_{2}(\mathrm{MA})_{n-1} \mathrm{~Pb}_{n} \mathrm{I}_{3 n+1}$ where $\mathrm{n}$ indicates the number of octahedron in the perovskite layer. These structures belong to the so-called Ruddlesden-Popper (RP) phases. ${ }^{17}$ (b) Computed band alignment for the RP HOP with $\mathrm{n}=1$ to 5. Experimental band gaps, extracted from continuum absorption thresholds, ${ }^{21}$ are used for conduction band lineup. (c) Hole (circle) and electron (square) confinement potentials extracted from panel $\mathrm{b}$ with corresponding ratios.

in the framework of the effective mass model, whose limitations will be assessed.

\section{Validity of the effective mass approximation}

To investigate the validity of the effective mass approximation (EMA) in predicting electronic properties, such as the band gap, we further consider the RP series (Figure 6a). For a 2D system modeled as a quantum well with finite confinement potentials, the band gap can be 
calculated using:

$$
E_{g}=E_{g}^{3 D}+E_{V B}+E_{C B}
$$

where $\mathrm{E}_{V B}$ and $\mathrm{E}_{C B}$ are ground state $\mathrm{VB}$ and $\mathrm{CB}$ energies respectively. Using the EMA with finite potential barriers, one obtains the following for the valence band: ${ }^{40}$

$$
\alpha=\frac{m_{V B}^{b}}{m_{V B}^{w}} k \tan \left(k \frac{L}{2}\right)
$$

where

$$
k=\sqrt{\frac{2 m_{V B}^{w} E_{V B}}{\hbar^{2}}} \text { and } \alpha=\sqrt{\frac{2 m_{V B}^{b}\left(V_{h}-E_{V B}\right)}{\hbar^{2}}}
$$

Here h is Planck's constant and materials parameters entering these equations are defined in Figure 1a. For conduction band ground state energy $\mathrm{E}_{C B}$, a similar Equation 4 can be written, where $\mathrm{V}_{h}$ and subscript $\mathrm{VB}$ are replaced with $\mathrm{V}_{e}$ and $\mathrm{CB}$, respectively.

Subsequently, Equations 4 and 5 can be solved numerically for $\mathrm{E}_{V B}$ and $\mathrm{E}_{C B}$ entering Equation 3. This requires knowledge of effective masses of holes $\left(\mathrm{m}_{h}\right)$ and electrons $\left(\mathrm{m}_{e}\right)$. These masses can be computed either in the plane parallel to the perovskite (well) layer $\left(\mathrm{m}_{\|}\right)$or along the stacking axis $\left(\mathrm{m}_{\perp}\right)$. From the composite approach, the pseudo-structures $\mathrm{W}$ and $\mathrm{B}$ are defined along the stacking axis (Figure 1b) and hence the effective masses in equation 4 are relevant to $\mathrm{m}_{\perp}$ for both holes and electrons. Nevertheless, the computation of effective masses presents some challenges. Indeed, it has been shown in HOP that accurate prediction of effective masses can only be achieved using self-consistent many-body and relativistic effects ${ }^{65}$ that are currently out-of-reach for the layered structures of our study. Hence, we use effective masses calibrated to a reduced mass of about $0.200 \mathrm{~m}_{0}$ as measured from magneto-absorption experiments ${ }^{66}$ as well as those used to fit the experimental band gaps (Table S5).

Next, for different $n$ values we employ the computed $V_{h}$ and $V_{e}$ (Figure $6 \mathrm{~b}$ ). Figure $7 \mathrm{a}$ 
a)

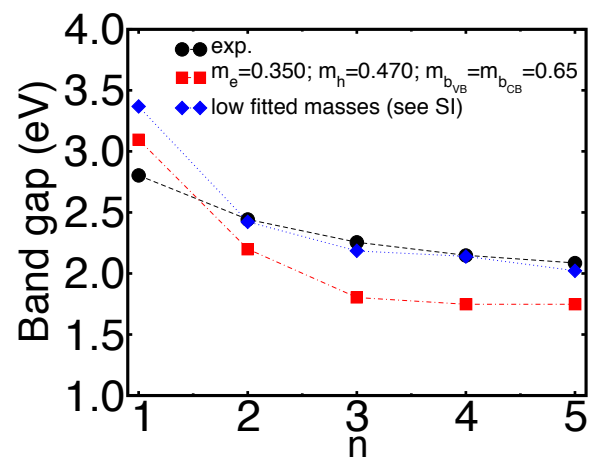

b)

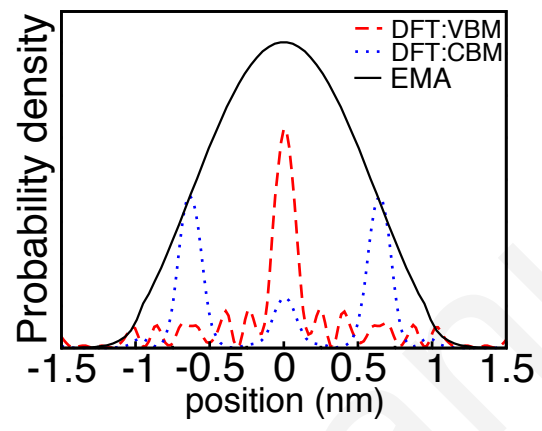

c)

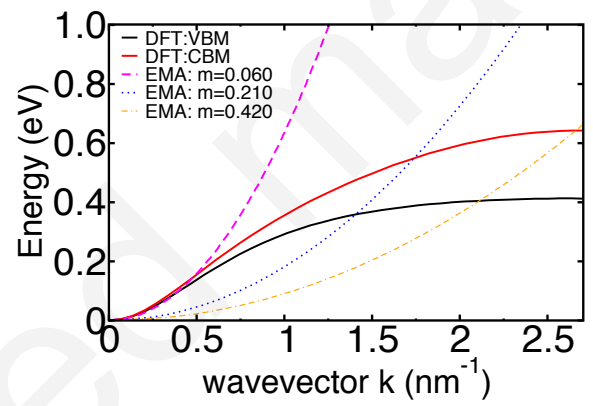

Figure 7: Limitations of the effective mass approximation when applied to layered HOP. (a) Experimental continuum absorption thresholds, band gaps, ${ }^{21}$ (black circles) compared to those calculated using the effective mass approximation (Eq. 2 and 3), considering either effective masses fitted so as to reproduce the experimentally determined reduced masses (red square) or significantly lower effective masses (Table S5) that fit reasonably experimental band gaps except for $\mathrm{n}=1$ (blue diamond). (b) Probability densities of VBM and CBM obtained within DFT compared to that computed for the $\mathrm{CB}$ within the effective mass approximation (For DFT, $\mathrm{n}=3$ from Figure 6 a was used). (c) Energy dispersion as derived within the effective mass appromixation (dashed-dotted lines) and DFT (straight-lines). DFT dispersion for both the CBM and the VBM evidences the non-parabolicity that cannot be captured in the effective mass approximation.

compares the experimental continuum band gaps ${ }^{21}$ with those deduced within the effective mass model. Using the effective masses calibrated to the experimental reduced ones $\left(\mathrm{m}_{e}=\right.$ $\left.0.350 \mathrm{~m}_{0}, \mathrm{~m}_{h}=0.470 \mathrm{~m}_{0}\right)$ fails to reproduce the experimental band gaps; however, lower 
values of effective masses $\left(\mathrm{m}_{e} \leq 0.105 \mathrm{~m}_{0}, \mathrm{~m}_{h} \leq 0.220 \mathrm{~m}_{0}\right)$ are able to recover them (see Table S5 for fitted masses). Nevertheless, these reduced masses are more than 3 times lower than the previous ones. In all cases, the effective mass approximation fails for $n=1 .{ }^{39,67}$ This is consistent with the above mentioned difference between the shift of $\mathrm{Pb} 5 \mathrm{~d}$ states and the Hartree potentials difference, showing that for $n=1$ the system is dominated by interface effects. Hence, the composite approach towards $n=1$ presents a larger error compared to the other $n$ members of $2 \mathrm{D}$ RP series $(\mathrm{n}=2-5)$.

In contrast to earlier work, ${ }^{67}$ the effective mass approximation cannot reproduce experimental band gaps for realistic reduced effective mass values even for larger wells ( $\mathrm{n}=2$ to 5 ). Thus, such approximation fails for this family of materials.

This failure can be understood through the absence of common Bloch functions between the inorganic well and the organic barrier, along the stacking axis, that is a basic assumption in quantum well models through the continuity equation across the boundaries. ${ }^{39}$ Besides, the probability density profiles computed using VBM and CBM wavefunctions from DFT are clearly different from the square of cosine probability density expected with the EMA (Figure $7 \mathrm{~b}$ ). As such, the envelope function approximation (another basic approach in semiconductor heterostructures within the EMA) is not valid in these 2D HOP structures. Finally, Figure 7c compares the energy dispersions of VBM and CBM obtained with DFT to the variation of the energy with respect to k-wavevector within the EMA. Only for k values lower than $0.5 \mathrm{~nm}^{-1}$, DFT based VBM and CBM vary reminiscent to the effective mass model when $0.06 \mathrm{~m}_{0}$ value is used for the latter. With realistic effective masses, DFT based dispersions cannot be reproduced by the effective mass model for any $\mathrm{k}$ values. This is a consequence of the non-parabolicity of the DFT dispersions along the stacking axis, which differs from the parabolicity assumption in the EMA. Therefore, first-principles methods are necessary to accurately describe opto-electronic properties of this family of materials. We stress, however, that there are systems (for instance, GaAs/AlAs heterostructure) for which the effective mass approximation performs reasonably well. ${ }^{68}$ 


\section{Design guidelines}

Having analyzed both the roles of material composition and structural properties on band alignment in 2D HOP, here we bring together a holistic view of the different effects aiming to formulate guidelines for device optimization. The major difference between type-I and type-II alignments is the spatial localization of holes and electrons. As such the type of 2D HOP alignment has a major influence on their excitonic properties. Resorting to 2D $\mathrm{HOP}$ containing only one inorganic layer with $\mathrm{Cl}$ as halogen can be good candidates for long-living excitons with enhanced emission properties due to its trending towards a possible type-II alignment. Such type-II alignment could be achieved by adopting organic barriers with narrower band gaps (by using, for example, conjugated moieties). The combination of 2D HOP with I as halogen and organic barriers with narrower band gaps may lead to lower confinement potentials in a type-I alignment, which is desirable for efficient carrier extraction from the perovskite layer. Moreover, self-assembled 2D HOPs with wider band gap molecular barriers potentially result in larger confinement potentials. Such systems with larger confinements could be interesting for optical confinement and may have potential application in guided optical emission devices as in edge light emitting diodes (ELED). ${ }^{69}$ The thickness of the perovskite slab along with the length of the organic barriers are also playground for materials engineering of these devices. Finally, tuning distorted structures through thermal processing or doping is a possible optimization route to optimize carrier confinement potentials and subsequently, charge transport or emission efficiency.

\section{Conclusions}

We presented a systematic approach that treats two dimensional hybrid organic-inorganic as composite materials. The approach evaluates band alignments of the layered HOP allowing access to carriers' confinement potentials. The latter depend on the composition and structural properties of the layered perovskites. In particular, monolayered $(n=1)$ HOPs 


\section{Methods}

The calculations were performed within the Density Functional Theory (DFT) ${ }^{70,71}$ as implemented in SIESTA package ${ }^{72}$ with a basis set of finite-range of numerical atomic orbitals. We used a van der Waals corrected functional with C09 exchange (VDW-C09) ${ }^{73}$ to describe the exchange-correlation term. Norm-conserving Troullier-Martins pseudopotentials were used for each atomic species to account for the core electrons. ${ }^{74} 1 \mathrm{~s}^{1}, 2 \mathrm{~s}^{2} 2 \mathrm{p}^{2}, 2 \mathrm{~s}^{2} 2 \mathrm{p}^{3}, 3 \mathrm{~s}^{2} 3 \mathrm{p}^{5}$, $4 \mathrm{~s}^{2} 4 \mathrm{p}^{5}, 5 \mathrm{~s}^{2} 5 \mathrm{p}^{5}$ and $5 \mathrm{~d}^{10} 6 \mathrm{~s}^{2} 6 \mathrm{p}^{2}$ were used as valence electrons for $\mathrm{H}, \mathrm{C}, \mathrm{N}, \mathrm{Cl}, \mathrm{Br}, \mathrm{I}$ and $\mathrm{Pb}$ respectively. Polarized Double-Zeta (DZP) basis set with an energy shift of $200 \mathrm{meV}$ and a real space mesh grid energy cutoff of 200 Rydberg were used for the calculations. The Brillouin zone was sampled with a $4 \times 4 \times 1$ Monckhorst-Pack grid. The electronic and dielectric properties were calculated using the experimental lattice parameters, and atomic coordinates were transformed to their primitive cells whenever applicable for their band 
structure computations. The used experimental structures were recorded at room temperature otherwise specified. Since the positions of H are not well resolved by X-Ray diffraction techniques, they were optimized within the primitive cells while keeping the other atomic positions fixed. Although its effect is significant in $\mathrm{Pb}$ based perovskites, spin-orbit coupling (SOC) was not taken into account in the band alignment procedures. This is because SOC effect is important at the conduction band maximum ${ }^{51}$ and band gaps are already underestimated within DFT. Hence, we focused our discussion on the valence band alignment. For the high-frequency dielectric constant profiles, slabs based on the respective systems were constructed, and an electric field of $0.01 \mathrm{eV} / \AA$ was applied along the [001] direction to compute the relaxed electron densities, as described elsewhere. ${ }^{45,46}$

\section{Acknowledgement}

We gratefully acknowledge Professor M. Koshimizu and Dr. N. Kawano for providing atomic positions of $(\mathrm{PMA})_{2} \mathrm{PbBr}_{4}$ from Ref. 15. The work at the Institut des Sciences Chimiques de Rennes was supported by Agence Nationale pour la Recherche (TRANSHYPERO project). This work was granted access to the HPC resources of [TGCC/CINES/IDRIS] under the allocation 2017-A0010907682 made by GENCI. ISCR and FOTON have received funding from the European Union's Horizon 2020 program, through a FET Open research and innovation action under the grant agreement No 687008. CCS and MGK acknowledge support from the Office of Naval Research (ONR grant N00014-17-1-2231). The work at Los Alamos National Laboratory (LANL) was supported by LANL LDRD program (J-C.B., H.T., W.N., S.T., A.D.M.) and was partially performed at the Center for Nonlinear Studies and at the Center for Integrated Nanotechnologies, a U.S. Department of Energy, Office of Science user facility. 


\section{Supporting Information Available}

The supplementary information file is available free of charge.

- The supplementary information contains: validity of substituting inter-layer molecules by Cs for states close to the band gap; Band alignments with DFT band gaps when the experimental band gaps are used in the main text; Definition of the octahedral tiltings; Tables containing octahedral titl angles for different structures $\left(\mathrm{C}_{m}, \mathrm{n}=1-5\right.$ 2D RP, $(\mathrm{PMA})_{2} \mathrm{PbX}_{4}, \mathrm{C} 10$ for phase transition effect); Note on band alignment for monolayered $(n=1)$ structures; Effective masses used to fit band gaps.

This material is available free of charge via the Internet at http://pubs.acs.org/.

\section{References}

1. Kojima, A.; Teshima, K.; Shirai, Y.; Miyasaka, T. Organometal Halide Perovskites as Visible-Light Sensitizers for Photovoltaic Cells. J.Am. Chem.Soc. 2009, 131, 60506051.

2. Im, J.-H.; Lee, C.-R.; Lee, J.-W.; Park, S.-W.; Park, N.-G. 6.5\% Efficient Perovskite Quantum-Dot-sensitized Solar Cell. Nanoscale 2011, 3, 4088-4093.

3. Lee, M. M.; Teuscher, J.; Miyasaka, T.; Murakami, T. N.; Snaith, H. J. Efficient Hybrid Solar Cells Based on Meso-Superstructured Organometal Halide Perovskites. Science 2012, 338, 643-647.

4. Kim, H.-S.; Lee, C.-R.; Im, J.-H.; Lee, K.-B.; Moehl, T.; Marchioro, A.; Moon, S.-J.; Humphry-Baker, R.; Yum, J.-H.; Moser, J. E. ; Grätzel, M. ; Park, N.-G. Lead Iodide Perovskite Sensitized All-Solid-State Submicron Thin Film Mesoscopic Solar Cell with Efficiency Exceeding 9\%. Sci.Ren. 2012, 2, 591. 
5. Tsai, H.; Nie, W.; Blancon, J.; Stoumpos, C.; Asadpour, R.; Harutyunyan, B.; Neukirch, A.; Verduzco, R.; Crochet, J.; Tretiak, S.; Pedesseau, L.; Even, J.; Alam, M. A.; Gautam, G.; Jun, L.; Ajayan, P. M.; and Bedzyk, M. J.; Kanatzidis, M. G.; Mohite, A. D. High-Efficiency Two-Dimensional Ruddlesden-Popper Perovskite Solar Cells. Nature 2016, 536, 312-317.

6. Yang, W. S.; Park, B.-W.; Jung, E. H.; Jeon, N. J.; Kim, Y. C.; Lee, D. U.; Shin, S. S.; Seo, J.; Kim, E. K.; Noh, J. H.; Seok, I. S. Iodide Management in Formamidinium-LeadHalide-Based Perovskite Layers for Efficient Solar Cells. Science 2017, 356, 1376-1379.

7. Even, J.; Pedesseau, L.; Katan, C. Analysis of Multivalley and Multibandgap Absorption and Enhancement of Free Carriers Related to Exciton Screening in Hybrid Perovskites. J. Phys. Chem. C 2014, 118, 11566-11572.

8. Amat, A.; Mosconi, E.; Ronca, E.; Quarti, C.; Umari, P.; Nazeeruddin, M. K.; Grätzel, M.; Angelis, F. D. Cation-Induced Band-Gap Tuning in Organohalide Perovskites: Interplay of Spin-Orbit Coupling and Octahedra Tilting. Nano Lett. 2014, 14, $3608-3616$.

9. Matsumoto, F.; Vorpahl, S. M.; Banks, J. Q.; Sengupta, E.; Ginger, D. S. Photodecomposition and Morphology Evolution of Organometal Halide Perovskite Solar Cells. J. Phys. Chem. C 2015, 119, 20810-20816.

10. Christians, J. A.; Miranda Herrera, P. A.; Kamat, P. V. Transformation of the Excited State and Photovoltaic Efficiency of $\mathrm{CH}_{3} \mathrm{NH}_{3} \mathrm{PbI}_{3}$ Perovskite upon Controlled Exposure to Humidified Air. J. Am. Chem. Soc. 2015, 13\%, 1530-1538.

11. Li, D.; Bretschneider, S. A.; Bergmann, V. W.; Hermes, I. M.; Mars, J.; Klasen, A.; Lu, H.; Tremel, W.; Mezger, M.; Butt, H.-J.; Weber, S. A. L.; Berger, R. HumidityInduced Grain Boundaries in $\mathrm{MAPbI}_{3}$ Perovskite Films. J. Phys. Chem. C 2016, 120, 6363-6368. 
12. Nie, W.; Blancon, J.-C.; Neukirch, A. J.; Appavoo, K.; Tsai, H.; Chhowalla, M.; Alam, M. A.; Sfeir, M. Y.; Katan, C.; Even, J.; Tretiak, S.; Crochet, J. J.; Gupta, G.; Mohite, A. D. Light-Activated Photocurrent Degradation and Self-Healing in Perovskite Solar Cells. Nat. Commun. 2016, \%, 11574.

13. Smith, I. C.; Hoke, E. T.; Solis-Ibarra, D.; McGehee, M. D.; Karunadasa, H. I. A Layered Hybrid Perovskite Solar-Cell Absorber with Enhanced Moisture Stability. Angew. Chem., Int. Ed. 2014, 53, 11232-11235.

14. Grancini, G.; Roldán-Carmona, C.; Zimmermann, I.; Mosconi, E.; Lee, X.; Martineau, D.; Narbey, S.; Oswald, F.; De Angelis, F.; Grätzel, M.; Nazeeruddin, M. K. One-Year Stable Perovskite Solar Cells by 2D/3D Interface Engineering. Nat. Commun. $2017,8,15684$.

15. Kawano, N.; Koshimizu, M.; Sun, Y.; Yahaba, N.; Fujimoto, Y.; Yanagida, T.; Asai, K. Effects of Organic Moieties on Luminescence Properties of Organic-Inorganic Layered Perovskite-Type Compounds. J. Phys. Chem. C 2014, 118, 9101-9106.

16. Smith, M. D.; Pedesseau, L.; Kepenekian, M.; Smith, I. C.; Katan, C.; Even, J.; Karunadasa, H. I. Decreasing the Electronic Confinement in Layered Perovskites Through Intercalation. Chem. Sci. 2017, 8, 1960-1968.

17. Stoumpos, C. C.; Cao, D. H.; Clark, D. J.; Young, J.; Rondinelli, J. M.; Jang, J. I.; Hupp, J. T.; Kanatzidis, M. G. Ruddlesden-Popper Hybrid Lead Iodide Perovskite 2D Homologous Semiconductors. Chem. Mater. 2016, 28, 2852-2867.

18. Stoumpos, C. C.; Soe, C. M. M.; Tsai, H.; Nie, W.; Blancon, J.-C.; Cao, D. H.; Liu, F.; Traoré, B.; Katan, C.; Even, J.; Aditya, D. M.; Mercouri, G. K. High Members of the 2D Ruddlesden-Popper Halide Perovskites: Synthesis, Optical Properties, and Solar Cells of $\left(\mathrm{CH}_{3}\left(\mathrm{CH}_{2}\right)_{3} \mathrm{NH}_{3}\right)_{2}\left(\mathrm{CH}_{3} \mathrm{NH}_{3}\right)_{4} \mathrm{~Pb}_{5} \mathrm{I}_{16}$. Chem 2017, 2, $427-440$. 
19. Vargas, B.; Ramos, E.; Pérez-Gutiérrez, E.; Alonso, J. C.; Solis-Ibarra, D. A Direct Bandgap Copper-Antimony Halide Perovskite. J. Am. Chem. Soc. 2017, 139, 91169119.

20. Tanaka, K.; Sano, F.; Takahashi, T.; Kondo, T.; Ito, R.; Ema, K. Two-dimensional Wannier Excitons in a Layered-Perovskite-Type Crystal $\left(\mathrm{C}_{6} \mathrm{H}_{13} \mathrm{NH}_{3}\right)_{2} \mathrm{PbI}_{4}$. Solid State Commun. 2002, 122, 249-252.

21. Blancon, J.-C.; Tsai, H.; Nie, W.; Stoumpos, C. C.; Pedesseau, L.; Katan, C.; Kepenekian, M.; Soe, C. M. M.; Appavoo, K.; Sfeir, M. Y.; Tretiak, S.; Ajayan, P. M.; Kanatzidis, M. G.; Even, J.; Crochet, J. J.; Mohite, A. D. Extremely Efficient Internal Exciton Dissociation Through Edge States in Layered 2D Perovskites. Science 2017, 335, 1288-1292.

22. Dohner, E. R.; Jaffe, A.; Bradshaw, L. R.; Karunadasa, H. I. Intrinsic White-Light Emission from Layered Hybrid Perovskites. J. Am. Chem. Soc. 2014, 136, 13154-13157.

23. Hu, T.; Smith, M. D.; Dohner, E. R.; Sher, M.-J.; Wu, X.; Trinh, M. T.; Fisher, A.; Corbett, J.; Zhu, X.-Y.; Karunadasa, H. I.; Lindenberg, A. M. Mechanism for Broadband White-Light Emission from Two-Dimensional (110) Hybrid Perovskites. J. Phys. Chem. Lett. 2016, ๆ, 2258-2263.

24. Mao, L.; Wu, Y.; Stoumpos, C. C.; Traore, B.; Katan, C.; Even, J.; Wasielewski, M. R.; Kanatzidis, M. G. Tunable White-Light Emission in Single-Cation-Templated ThreeLayered 2D Perovskites $\left(\mathrm{CH}_{3} \mathrm{CH}_{2} \mathrm{NH}_{3}\right)_{4} \mathrm{~Pb}_{3} \mathrm{Br}_{10 x} \mathrm{Cl}_{x}$. J. Am. Chem. Soc. 2017, 139, $11956-11963$.

25. Hong, X.; Ishihara, T.; Nurmikko, A. V. Dielectric Confinement Effect on Excitons in $\mathrm{PbI}_{4}$-based Layered Semiconductors. Phys. Rev. B 1992, 45, 6961-6964.

26. Kato, Y.; Ichii, D.; Ohashi, K.; Kunugita, H.; Ema, K.; Tanaka, K.; Takahashi, T.; 
Kondo, T. Extremely Large Binding Energy of Biexcitons in an Organic-Inorganic Quantum-Well Material $\left(\mathrm{C}_{4} \mathrm{H}_{9} \mathrm{NH}_{3}\right)_{2} \mathrm{PbBr}_{4}$. Solid State Commun. 2003, 128, 15 -18.

27. Knutson, J. L.; ; Martin, J. D.; Mitzi, D. B. Tuning the Band Gap in Hybrid Tin Iodide Perovskite Semiconductors Using Structural Templating. Inorg. Chem. 2005, 44, 4699-4705.

28. Zhou, Z.; Pang, S.; Liu, Z.; Xu, H.; Cui, G. Interface Engineering for High-Performance Perovskite Hybrid Solar Cells. J. Mater. Chem. A 2015, 3, 19205-19217.

29. Hays, D. C.; Gila, B. P.; Pearton, S. J.; Ren, F. Energy Band Offsets of Dielectrics on InGaZnO. . Appl. Phys. Rev. 2017, 4, 021301.

30. Kleider, J.-P. In Physics and Technology of Amorphous-Crystalline Heterostructure Silicon Solar Cells; van Sark, W. G. J. H. M., Korte, L., Roca, F., Eds.; Springer Berlin Heidelberg: Berlin, Heidelberg, 2012; pp 405-444.

31. Anderson, R. L. Experiments on Ge-GaAs heterojunctions. Solid State Electron. 1962, $5,341-351$.

32. Nakagawa, A.; Kroemer, H.; English, J. H. Electrical Properties and Band Offsets of InAs/AlSb n-N Isotype Heterojunctions Grown on GaAs. Appl. Phys. Lett. 1989, 54, $1893-1895$.

33. Waldrop, J. R.; Kraut, E. A.; Farley, C. W.; Grant, R. W. Measurement of $\operatorname{InP} / \operatorname{In}_{0.53} \mathrm{Ga}_{0.47} \mathrm{As}$ and $\operatorname{In}_{0.53} \mathrm{Ga}_{0.47} \mathrm{As} / \mathrm{In}_{0.52} \mathrm{Al}_{0.48} \mathrm{As}$ Heterojunction Band Offsets by XRay Photoemission Spectroscopy. J. Appl. Phys. 1991, 69, 372-378.

34. Van de Walle, C. G.; Martin, R. M. Theoretical Study of Band Offsets at Semiconductor Interfaces. Phys. Rev. B 1987, 35, 8154-8165.

35. Baldereschi, A.; Baroni, S.; Resta, R. Band Offsets in Lattice-Matched Heterojunctions: 
A Model and First-Principles Calculations for GaAs/AlAs. Phys. Rev. Lett. 1988, 61, $734-737$.

36. Junquera, J.; Zimmer, M.; Ordejón, P.; Ghosez, P. First-principles Calculation of The Band Offset at $\mathrm{BaO} / \mathrm{BaTiO}_{3}$ and $\mathrm{SrO} / \mathrm{SrTiO}_{3}$ Interfaces. Phys. Rev. B 2003, 67, 155327.

37. Kleinman, L. Comment on The Average Potential of a Wigner Solid. Phys. Rev. B 1981, 24, 7412-7414.

38. Mitzi, D. B.; Chondroudis, K.; Kagan, C. R. Organic-Inorganic Electronics. IBM J. Res. Dev. 2001, 45, 29-45.

39. Even, J.; Pedesseau, L.; Katan, C. Understanding Quantum Confinement of Charge Carriers in Layered 2D Hybrid Perovskites. ChemPhysChem 2014, 15, 3733-3741.

40. Chuang, S. Physics of Optoelectronic Devices; Wiley Series in Pure and Applied Optics; Wiley, 1995.

41. Keldysh, L. V. Coulomb Interaction in Thin Semiconductor and Semimetal Films. JETP Lett. 1979, 29, 658.

42. Kumagai, M.; Takagahara, T. Excitonic and Nonlinear-Optical Properties of Dielectric Quantum-Well Structures. Phys. Rev. B 1989, 40, 12359-12381.

43. Giustino, F.; Pasquarello, A. Theory of Atomic-Scale Dielectric Permittivity at Insulator Interfaces. Phys. Rev. B 2005, 71, 144104.

44. Shi, N.; Ramprasad, R. Atomic-Scale Dielectric Permittivity Profiles in Slabs and Multilayers. Phys. Rev. B 2006, 74, 045318.

45. Even, J.; Pedesseau, L.; Kepenekian, M. Electronic Surface States and Dielectric SelfEnergy Profiles in Colloidal Nanoscale Platelets of CdSe. Phys. Chem. Chem. Phys. 2014, 16, 25182-25190. 
48. Lee, K.-S.; Kang, R.; Son, B.; Kim, D.-Y.; Yu, N. E.; Ko, D.-K. All-Optical THz Wave Switching Based on $\mathrm{CH}_{3} \mathrm{NH}_{3} \mathrm{PbI}_{3}$ perovskites. Sci. Rep. 2016, 6, 37912, Article.

49. Miyata, K.; Atallah, T. L.; Zhu, X.-Y. Lead Halide Perovskites: Crystal-Liquid Duality, Phonon Glass Electron Crystals, and Large Polaron Formation. Sci. Adv. 2017, 3.

50. Chanana, A.; Zhai, Y.; Baniya, S.; Zhang, C.; Vardeny, Z. V.; Nahata, A. Colour Selective Control of Terahertz Radiation Using Two-Dimensional Hybrid Organic Inorganic Lead-Trihalide Perovskites. Nat. Commun. 2017, 8, 1328.

51. Even, J.; Pedesseau, L.; Jancu, J.-M.; Katan, C. Importance of Spin-Orbit Coupling in Hybrid Organic/Inorganic Perovskites for Photovoltaic Applications. J. Phys. Chem. Lett. 2013, 4, 2999-3005.

52. Katan, C.; Pedesseau, L.; Kepenekian, M.; Rolland, A.; Even, J. Interplay of SpinOrbit Coupling and Lattice Distortion in Metal Substituted 3D Tri-Chloride Hybrid Perovskites. J. Mater. Chem. A 2015, 3, 9232-9240.

53. Zhang, S.; Audebert, P.; Wei, Y.; Al Choueiry, A.; Lanty, G.;Bréhier, A.; Galmiche, L.; Clavier, G.; Boissiere, C.; Lauret, J.-S.; Deleporte, E. Preparations and Characterizations of Luminescent Two Dimensional Organic-Inorganic Perovskite Semiconductors. Materials 2010, 3, 3385-3406. 
54. Shi, D.; Adinolfi, V.; Comin, R.; Yuan, M.; Alarousu, E.; Buin, A.; Chen, Y.; Hoogland, S.; Rothenberger, A.; Katsiev, K.; Losovyj, Y.; Zhang, X.; Dowben, P. A.; Mohammed, O. F.; Sargent, E. H.; Bakr, O. M. Low Trap-State Density and Long Carrier Diffusion in Organolead Trihalide Perovskite Single Crystals. Science 2015, 347, 519522 .

55. Papavassiliou, G. C.; Mousdis, G. I.; Raptopoulou, C. P.; Terzis, A. Preparation and Characterization of $\left[\mathrm{C}_{6} \mathrm{H}_{5} \mathrm{CH}_{2} \mathrm{NH}_{3}\right]_{2} \mathrm{PbI}_{4},\left[\mathrm{C}_{6} \mathrm{H}_{5} \mathrm{CH}_{2} \mathrm{CH}_{2} \mathrm{SC}\left(\mathrm{NH}_{2}\right)_{2}\right]_{3} \mathrm{PbI}_{5}$, and $\left[\mathrm{C}_{10} \mathrm{H}_{7} \mathrm{CH}_{2} \mathrm{NH}_{3}\right] \mathrm{PbI}_{3}$ Organic-Inorganic Hybrid Compounds. Z. Naturforsch. 1999, 54b, 1405 .

56. Liao, W.-Q.; Zhang, Y.; Hu, C.-L.; Mao, J.-G.; Ye, H.-Y.; Li, P.-F.; Huang, S. D.; Xiong, R.-G. A Lead-Halide Perovskite Molecular Ferroelectric Semiconductor. Nat. Commun. 2015, 6, 1-7.

57. Billing, D. G.; Lemmerer, A. Synthesis, Characterization and Phase Transitions of The Inorganic-Organic Layered Perovskite-Type Hybrids $\left[\left(\mathrm{C}_{n} \mathrm{H}_{2 n+1} \mathrm{NH}_{3}\right)_{2} \mathrm{PbI}_{4}\right](\mathrm{n}=12,14$, 16 and 18). New J. Chem. 2008, 32, 1736-1746.

58. Mitzi, D. B. Synthesis, Crystal Structure, and Optical and Thermal Properties of $\left(\mathrm{C}_{4} \mathrm{H}_{9} \mathrm{NH}_{3}\right)_{2} \mathrm{MI}_{4}(\mathrm{M}=\mathrm{Ge}, \mathrm{Sn}, \mathrm{Pb})$. Chem. Mater. 1996, 8, 791-800.

59. Cao, D. H.; Stoumpos, C. C.; Yokoyama, T.; Logsdon, J. L.; Song, T.-B.; Farha, O. K.; Wasielewski, M. R.; Hupp, J. T.; Kanatzidis, M. G. Thin Films and Solar Cells Based on Semiconducting Two-Dimensional Ruddlesden-Popper $\left(\mathrm{CH}_{3}\left(\mathrm{CH}_{2}\right)_{3} \mathrm{NH}_{3}\right)_{2}\left(\mathrm{CH}_{3} \mathrm{NH}_{3}\right)_{n-1} \mathrm{Sn}_{n} \mathrm{I}_{3 n+1}$ Perovskites. ACS Energy Lett. 2017, 2, 982990 .

60. Pedesseau, L.; Sapori, D.; Traore, B.; Robles, R.; Fang, H.-H.; Loi, M. A.; Tsai, H.; Nie, W.; Blancon, J.-C.; Neukirch, A.; Tretiak, S.; Mohite, A. D.; Katan, C.; Even, J.; 
Kepenekian, M. Advances and Promises of Layered Halide Hybrid Perovskite Semiconductors. ACS Nano 2016, 10, 9776-9786.

61. Billing, D. G.; Lemmerer, A. Synthesis, Characterization and Phase Tansitions of The Inorganic-Organic Layered Perovskite-Type Hybrids $\left[\left(\mathrm{C}_{n} \mathrm{H}_{2 n+1} \mathrm{NH}_{3}\right)_{2} \mathrm{PbI}_{4}\right](\mathrm{n}=12,14$, 16 and 18). New J. Chem. 2008, 32, 1736-1746.

62. Lemmerer, A.; Billing, D. G. Synthesis, Characterization and Phase Transitions of The Inorganic-Organic Layered Perovskite-Type Hybrids $\left[\left(\mathrm{C}_{n} \mathrm{H}_{2 n+1} \mathrm{NH}_{3}\right)_{2} \mathrm{PbI}_{4}\right], \mathrm{n}=7,8,9$ and 10. Dalton Trans. 2012, 41, 1146-1157.

63. Filip, M. R.; Eperon, G. E.; Snaith, H. J.; Giustino, F. Steric Engineering of Metal-Halide Perovskites With Tunable Optical Band Gaps. Nat. Commun. 2014, 5, 5757.

64. Chen, Y.; Sun, Y.; Peng, J.; Tang, J.; Zheng, K.; Liang, Z. 2D Ruddlesden-Popper Perovskites for Optoelectronics. Adv. Mater. 1703487.

65. Even, J.; Pedesseau, L.; Jancu, J.-M.; Katan, C. DFT and k.p Modelling of The Phase Transitions of Lead and Tin halide Perovskites for Photovoltaic Cells. Phys. Status Solidi RRL 2014, 8, 31-35.

66. Blancon, J.-C.; Stier, A. V.; Tsai, H.; Nie, W.; Stoumpos, C. C.; Traoré, B.; Pedesseau, L.; Kepenekian, M.; Tretiak, S.; Crooker, S. A.; Katan, C.; Kanatzidis, M. G.; Crochet, J. J.; Even, J.; Mohite, A. D. Unusual Thickness Dependence of Exciton Characteristics in 2D Perovskite Quantum Wells. ArXiv e-prints 2017,

67. K.Tanaka,; Takahashi, T.; Kondo, T.; Umeda, K.; Ema, K.; Umebayashi, T.; Asai, K.; Uchida, K.; Miura, N. Electronic and Excitonic Structures of Inorganic-Organic Perovskite-Type Quantum-Well Crystal $\left(\mathrm{C}_{4} \mathrm{H}_{9} \mathrm{NH}_{3}\right)_{2} \mathrm{PbBr}_{4}$. Jpn. J. Appl. Phys. 2005, 44, 5923 . 
68. Boyer-Richard, S.; Raouafi, F.; Bondi, A.; Pédesseau, L.; Katan, C.; Jancu, J.-M.; Even, J. 30-band k.p method for quantum semiconductor heterostructures. Appl. Phys. Lett. 2011, 98, 251913.

69. Vijayachitra, S. Communication Engineering; McGraw Hill Education (India), 2013.

70. Hohenberg, P.; Kohn, W. Inhomogeneous Electron Gas. Phys. Rev. 1964, 136, B864B871.

71. Kohn, W.; Sham, L. J. Self-Consistent Equations Including Exchange and Correlation Effects. Phys. Rev. 1965, 140, A1133-A1138.

72. Soler, M.; Artacho, E.; Gale, J. D.; Garc, A.; Junquera, J.; Ordejon, P.; Daniel, S. The SIESTA Method for Ab Initio Order-N Materials. J. Phys.: Condens. Matter 2002, 14, 2745 .

73. Cooper, V. R. Van der Waals Density Functional: An Appropriate Exchange Functional. Phys. Rev. B 2010, 81, 161104.

74. Troullier, N.; Martins, J. L. Efficient Pseudopotentials for Plane-Wave Calculations. Phys. Rev. B 1991, 43, 1993-2006. 


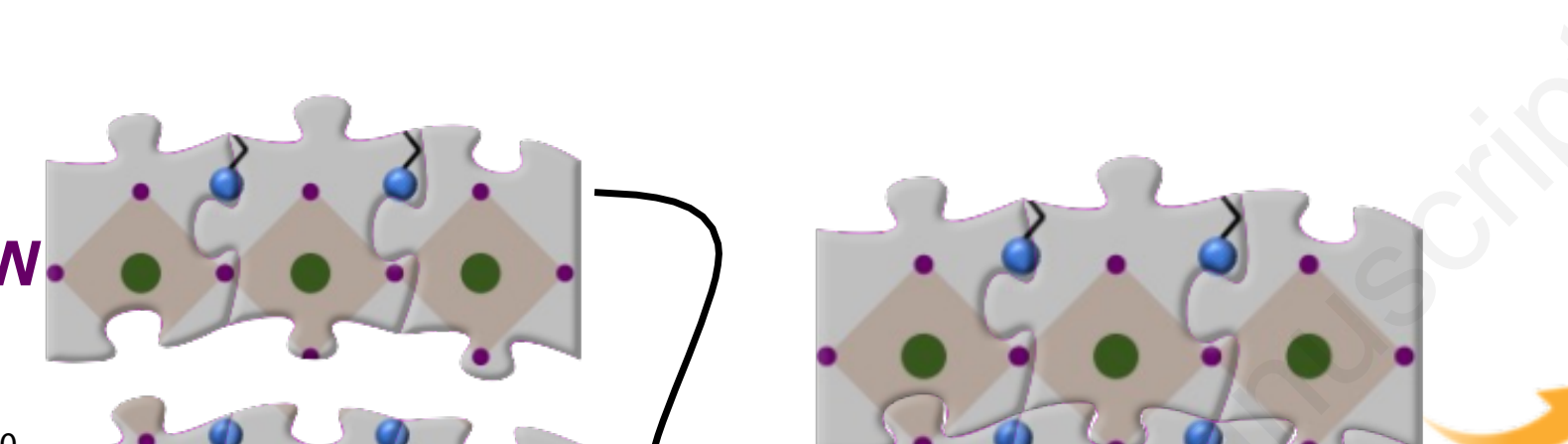

\section{Band alignment}

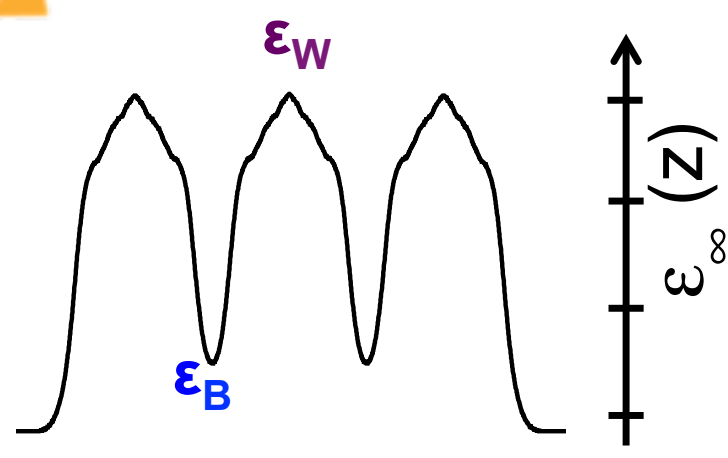

Dielectric contrast 